\title{
Joint Power Control and Scheduling for Two-Cell Energy Efficient Broadcasting with Network Coding
}

\author{
Linyu Huang ${ }^{1}$, Chi Wan Sung ${ }^{1}$, Seong-Lyun Kim ${ }^{2}$ \\ ${ }^{1}$ Dept. of Electronic Engineering, City University of Hong Kong, Hong Kong SAR \\ ${ }^{2}$ School of Electrical and Electronic Engineering, Yonsei University, Seoul, Korea \\ Email: L.huang@my.cityu.edu.hk, albert.sung@cityu.edu.hk, slkim@ramo.yonsei.ac.kr
}

Received July, 2013

\begin{abstract}
We consider the energy minimization problem for a two-cell broadcasting system, where the focus is devising energy efficient joint power control and scheduling algorithms. To improve the retransmission efficiency, linear network coding is applied to broadcast packets. Combined with network coding, an optimal algorithm is proposed, which is based on dynamic programming. To reduce computational complexity, two sub-optimal algorithms are also proposed for large networks. Simulation results show that the proposed schemes can reduce energy consumption up to $57 \%$ compared with the traditional Automatic Repeat-reQuest (ARQ).
\end{abstract}

Keywords: Power Control; Network Coding; Broadcast; Scheduling; Energy Minimization

\section{Introduction}

Energy efficiency is an important concern in wireless systems, both for environmental and economical reasons. The information and communication technology (ICT) infrastructure consumes about $3 \%$ of the world-wide energy and the $\mathrm{CO}_{2}$ emissions are as many as one quarter of the $\mathrm{CO}_{2}$ emissions by cars [1]. The $\mathrm{CO}_{2}$ emissions from ICT are still increasing at a rate of $6 \%$ per year [2]. Besides, the energy bill accounts for a large proportion in the costs of running a network. The operating costs can be significantly reduced by reducing energy consumption [3]. Therefore, energy efficiency is an increasingly important issue. In this paper, we focus on transmit energy minimization for a wireless broadcasting system consisting of two cells.

Linear network coding [4] can be used to reduce energy consumption by improving the retransmission efficiency. The traditional way to retransmit the lost packets is using Automatic Repeat-request (ARQ). The retransmitted packets may not be useful to every user, and therefore the traditional ARQ is energy inefficient. The promising network coding technique [4] has been proved to be an efficient approach for multi-receiver broadcast. Each encoded packet is a linear combination of original packets, where the combination coefficients are drawn from a certain finite field. These coefficients for each packet are grouped together, which is the encoding vector of that packet. The encoding vectors are broadcast together with the corresponding packets to all the receiv- ers. An encoding vector is said to be innovative to a receiver if it is not in the subspace spanned by the previously received encoding vectors of that user. An encoding vector is called innovative if it is innovative to all the receivers that have not received enough packets for decoding. For easier reading, we say that a packet is innovative if its corresponding encoding vector is innovative. Network coding can be applied into broadcasting systems to improve the retransmission performance.

There are some previous works that apply network coding to wireless broadcasting systems. For example, XOR network coding and random linear network coding (RLNC) are applied to wireless broadcast in [5] and [6], respectively. However, XOR operates in the binary field and innovative vectors cannot be guaranteed when there are more than two receivers. Although RLNC can provide innovative vectors when the finite field is sufficiently large, the large field size increases the computational complexities for encoding and decoding. In [7-8], Kwan et al. propose different coding methods which can guarantee innovative encoding vectors while have a relatively low requirement on field size. Their methods only require that the field size is no less the total number of receivers. We will use the method of [8] to improve the retransmission efficiency.

Another way to reduce energy consumption is to control the transmit power of base stations. By controlling the transmit power, we can control the received Signal to Interference plus Noise Ratio (SINR), so as to reduce energy consumption. To our best knowledge, most of the 
previous works on energy minimization for broadcasting are based on power control only [9] (and the references therein) [10], without using network coding. Although Tran et al. consider both power control and network coding in [5], their network coding method works over $G F(2)$ which cannot guarantee that all the encoding vectors are innovative. Note that both our power control scheme and network coding method are different from those in [5].

In this paper, we combine power control together with network coding for broadcasting networks. We focus on the joint power control and scheduling problem for two base stations. The objective is to minimize the expected transmit energy consumption. We use the network coding method proposed in [8] to generate innovative packets for transmissions. Combined with network coding, we propose three joint power control and scheduling algorithms to minimize energy consumption. In the first algorithm, the problem is formulated as a dynamic programming problem, which can provide an optimal solution, in the sense of minimizing the expected energy consumption. To reduce the computational complexity, two heuristic algorithms are also proposed for large networks. Simulation shows that they perform very well.

\section{System Model}

As shown in Figure 1, we consider a time slotted wireless broadcast system consisting of two partially overlapped cells and $K$ users. The two base stations are denoted by $B S_{1}$ and $B S_{2}$, respectively. The transmission range of each base station is a circle with radius $r$. The distance between the two base stations is denoted by $D$. We define a parameter $\delta=D / r$, where $0<\delta<2$, to characterize the locations of the two cells. The $K$ users are randomly located in the two cells and labeled as $U_{i}$, where $i \in\{1,2, \ldots, K\}$. The distance from $B S_{b}$, where $b$ $\in\{1,2\}$, to user $U_{i}$ is denoted by $d_{b i}$. A broadcast file is divided into $N$ equal-size packets which are called $u n$ coded packets. Both $B S_{1}$ and $B S_{2}$ have all the $N$ uncoded packets. They want to broadcast these $N$ packets to all the $K$ users by cooperating with each other.

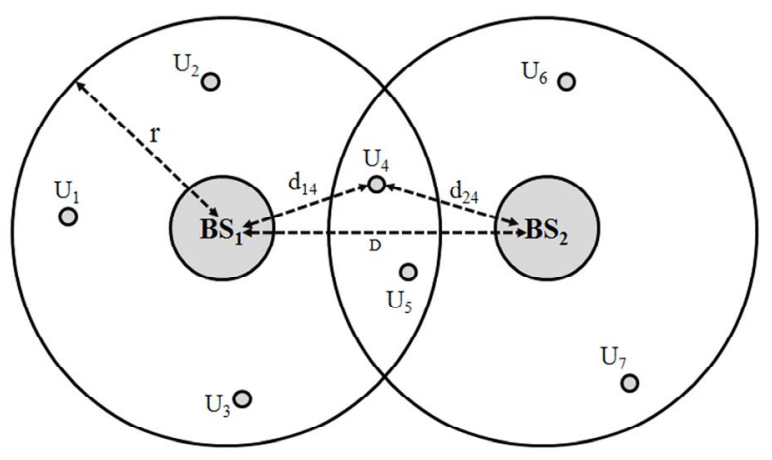

Figure 1. System model.
Both $B S_{1}$ and $B S_{2}$ can transmit to the users in their own transmission range. To avoid collisions, they are not allowed to transmit simultaneously. At each time slot, both $B S_{1}$ and $B S_{2}$ can change its transmit power to a value which is chosen from a preset power level collection $\Theta=$ $\left\{\theta_{1}, \theta_{2}, \ldots, \theta_{|\Theta|},\right\}$ (in $\mathrm{mW}$ units). All downlink channels are modeled as mutually independent channels, across time and across users, which are affected by path loss and Rayleigh fading. A packet is considered to be successfully received if the received SINR is greater than a predetermined threshold $\Gamma$ (in $\mathrm{dB}$ units). Otherwise, that packet will be considered to be lost. We use $R_{b i}\left(\theta_{m}\right)$ to denote the reliability of the downlink channel from $B S_{b}$ to user $U_{i}$ with transmit power $\theta_{m}$. The reliability is defined as the probability that the received SINR is greater than the threshold $\Gamma$. Since the received signal is subject to Rayleigh fading, the instantaneous SINR is distributed according to an exponential distribution with parameter $1 / \bar{S}$ [11], where $\bar{S}$ is the average SINR. According to the cumulative distribution function of exponential distribution, the reliability $R_{b i}\left(\theta_{m}\right)$ can be computed by

$$
R_{b i}\left(\theta_{m}\right)=P\left\{S_{r}>\Gamma\right\}=\exp \left(-\frac{\Gamma}{\bar{S}}\right),
$$

where $S_{r}$ is the instantaneous received SINR and $\bar{S}$ is the average SINR (in dB units). Note that $\bar{S}$ is determined by the corresponding distance $d_{b i}$ and transmit power $\theta_{m}$. Depending on the environment, $S$ can be obtained by using the corresponding radio propagation model, such as the Okumura model and Hata model.

Once a user successfully received a packet, it will send an acknowledgement to the sender of that packet. All the acknowledgement channels are assumed to be reliable channels without error and delay. $B S_{1}$ and $B S_{2}$ are also assumed to be connected via a wired cable and they can share the acknowledgement information with each other.

All the packets transmitted by the base stations are coded packets based on linear network coding. We assume that the coding is operated over a finite field with $q$ elements, $G F(q)$, where $q \geq K$. We use the network coding method proposed in [8]. Since $q \geq K$, it is guaranteed that each broadcast packet is innovative to all users. In other words, a user can recover the original file as long as it has successfully received any $N$ broadcast packets. An example of network coding is given in Example 1. For more details about the network coding method, we refer the reader to [8]. We say that $U_{i}$ is complete if it has received $N$ broadcast packets. Otherwise, we say that $U_{i}$ is incomplete.

Example 1: Let $q=3, K=3$ and $N=3$. We assume that each encoding vector is a $1 \times 3$ row vector. For example, an encoding vector [ $\left[\begin{array}{lll}1 & 0 & 1\end{array}\right]$ means that the corresponding encoded packet is obtained by taking linear combinations of the first and the third uncoded packets 
with coefficients being 1 .

Let $\mathbf{C}_{i}$ be the encoding matrix of user $U_{i}$, where its rows represent the encoding vectors $U_{i}$ has received so far. At a particular time slot, consider

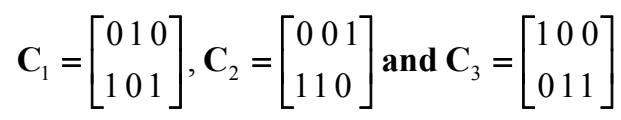

Each user needs to receive at least one more packet for successfully decoding the original file. If the network coding method proposed in [8] is used, we can broadcast an encoding vector $\mathbf{v}^{*}=\left[\begin{array}{lll}0 & 1 & 2\end{array}\right]$ to all users in one time slot. For each $U_{i}, \mathbf{v}^{*}$ is not in the row space of $\mathbf{C}_{i}$, i.e. $\mathbf{v}^{*}$ cannot be obtained by taking linear combinations of row vectors in $\mathbf{C}_{i}$ over $G F(3)$. Therefore, $\mathbf{v}^{*}$ is innovative to all the three users. As long as $U_{i}$ can successfully receive $\mathbf{v}^{*}$, it can get a full rank matrix by appending $\mathbf{v}^{*}$ to the end of $\mathbf{C}_{i}$. Then, $U_{i}$ can successfully decode the original file by using Gaussian elimination to solve the matrix equations.

However, if XOR network coding is used, it cannot find such an encoding vector that is innovative to all the three users simultaneously. It requires at least two transmissions. For example, the base station may transmit the encoding vector $\left[\begin{array}{lll}0 & 1 & 1\end{array}\right]$ to $U_{1}$ and $U_{2}$ in the first slot and the encoding vector $\left[\begin{array}{lll}0 & 1 & 0\end{array}\right]$ to $U_{3}$ in the second slot. The network coding method proposed in [8] outweighs XOR in this case.

Given a scheduling scheme $\mathbf{B}$, we use an integer random variable $T_{\mathbf{B}}$ to denote the required number of transmissions for $\mathbf{B}$ to complete the broadcast. We define the broadcast schedule of $\mathbf{B}$ as a binary sequence, $\Phi_{\mathbf{B}}=\left\{\varphi_{1}\right.$, $\left.\varphi_{2}, \ldots, \varphi_{T \mathrm{~B}}\right\}$. If $\varphi_{t}=0, B S_{1}$ is selected to transmit at the $t$-th time slot. Otherwise, if $\varphi_{t}=1, B S_{2}$ is selected. We define the sequence of transmit power $\Lambda_{\mathbf{B}}$ as $\left\{\lambda_{1}, \lambda_{2}, \ldots\right.$, $\left.\lambda_{T \mathbf{B}}\right\}$, where $\lambda_{t} \in \Theta$ is the transmit power level used at the $t$-th time slot.

At time $t, \mathbf{B}$ specifies a function $f_{t}$ to determine $\varphi_{t}$ and $\lambda_{t}$ :

$$
\left(\phi_{t}, \lambda_{t}\right)=f_{t}\left(\phi_{1}, \lambda_{1}, \mathbf{e}_{1}, \phi_{2}, \lambda_{2}, \mathbf{e}_{2}, \ldots, \phi_{t-1}, \lambda_{t-1}, \mathbf{e}_{t-1}\right)
$$

where $\mathbf{e}_{t}$ is the channel status at the $t$-th time slot. It is a binary random vector of length $K$. If $\mathbf{e}_{t}(i)=1$, it means that the channel between the transmitter and $U_{i}$ is too bad and $U_{i}$ cannot receive the broadcast packet. Otherwise, $U_{i}$ can receive that packet. Note that the probability distribution of $\mathbf{e}_{t}$ depends on whether $B S_{1}$ or $B S_{2}$ is chosen as the transmitter at time $t$. We call a realization of the sequence $\left\{\mathbf{e}_{1}, \mathbf{e}_{2}, \ldots, \mathbf{e}_{T \mathbf{B}}\right\}$ a channel realization under $\mathbf{B}$, denoted by $\Omega_{\mathbf{B}}$.

For a transmission with transmit power $\lambda_{t}$, the energy consumption is $\lambda_{t}$ times the duration of a time slot. To simplify notations, we assume that the duration is one unit and will be ignored throughout this paper. Therefore, the total energy consumption of a particular channel re- alization $\Omega_{\mathbf{B}}$ is denoted by $X_{\mathbf{B}}$ and can be computed as

$$
X_{\mathbf{B}}=\sum_{t=1}^{T_{\mathbf{B}}} \lambda_{t} \text {. }
$$

The expected energy consumption of $\mathbf{B}$ is denoted by $E\left[X_{\mathbf{B}}\right]$, where the expectation is taken with respect to the probability distribution of $\mathbf{e}_{t}$, which depends on $\varphi_{t}$. Our objective is to minimize $E\left[X_{\mathbf{B}}\right]$ by determining an optimal scheduling scheme $\mathbf{B}$ which requires to determine $\Phi_{\mathbf{B}}$ and $\Lambda_{\mathbf{B}}$ as well.

\section{Optimal Scheme}

The joint power control and scheduling problem can be formulated as a dynamic programming problem with the following parameters.

- State We define the state of all users as a $1 \times K$ state vector $\mathbf{s}=\left[\begin{array}{llll}s_{1} & s_{2} & \ldots & s_{K}\end{array}\right]$, where $s_{i}, 0<i \leq \mathrm{K}$, denotes the number of packets that $U_{i}$ has received. The state space is denoted by $\mathbf{S}=\left[\begin{array}{llll}s_{1} & s_{2} & \ldots & s_{K}\end{array}\right]$, where $0 \leq s_{i} \leq N$ and $0<$ $i \leq K$. The goal state is defined as $\mathbf{s}_{\mathrm{g}}=[N N \ldots N]$, which means every user has received $N$ packets and can be considered as a complete user.

- Action At each time step, we need to determine both the transmitter and the transmit power. We define the action set as $\mathbf{A}=\{0,1\} \times \Theta$. At each time slot, an action $a=(b, \theta)$ is selected from A. Action $a=\left(b, \theta_{m}\right)$ means that $B S_{b}$ is selected to transmit with transmit power $\theta_{m}$.

- State Transition Probability Given a state $\mathbf{s}$, let $p\left(\mathbf{s}^{\prime} \mid \mathbf{s}, a\right)$ denote the state transition probability that transfers from $\mathbf{s}$ to $\mathbf{s}^{\prime}$ under action $a$. Now, we are going to introduce how to compute the state transition probability $p(\mathbf{s} \mid \mathbf{s}, a)$.

For a given action $a=\left(b, \theta_{m}\right)$ and given states $\mathbf{s}=\left[s_{1}\right.$ $\left.s_{2} \ldots s_{K}\right]$ and $\mathbf{s}^{\prime}=\left[\begin{array}{lll}s_{1}{ }^{\prime} & s_{2}{ }^{\prime} \ldots & s_{K}{ }^{\prime}\end{array}\right]$, we use $p\left(s_{i}{ }^{\prime} \mid s_{i}, a\right)$ to denote the probability that the number of packets received by $U_{i}$ changes from $s_{i}$ to $s_{i}{ }^{\prime}$ under action $a$. Since all the downlink channels are assumed to be mutually independent across users, the state transition probability $p\left(\mathbf{s}^{\prime} \mid \mathbf{s}\right.$, $a)$ is the product of $p\left(s_{i}^{\prime} \mid s_{i}, a\right)$ for all users. Hence,

$$
p\left(\mathbf{s}^{\prime} \mid \mathbf{s}, a\right)=\prod_{i=1}^{K} p\left(s_{i}^{\prime} \mid s_{i}, a\right)
$$

We define $Q_{i}(a)$ as the success probability of taking action $a$ to transmit to user $U_{i}$. Based on the previous discussion, $Q_{i}(a)$ equals the reliability $R_{b i}\left(\theta_{m}\right)$ and $Q_{i}(a)$ can be obtained by Equation (1). Based on the given states $\mathbf{s}$, $\mathbf{s}^{\prime}$ and the number of packets that $U_{i}$ has received, the state transition for $U_{i}$ can be classified into five different cases. The transition probability of each case can be obtained by the following equation. 


$$
p\left(s_{i}{ }^{\prime} \mid s_{i}, a\right)= \begin{cases}0, & \text { if } s_{i}{ }^{\prime}<s_{i}, \\ 1, & \text { if } s_{i}{ }^{\prime}=s_{i}=N, \\ 1-Q_{i}(a), & \text { if } s_{i}{ }^{\prime}=s_{i}<N, \\ Q_{i}(a), & \text { if } s_{i}{ }^{\prime}-s_{i}=1, \\ 0, & \text { if } s_{i}{ }^{\prime}-s_{i} \geq 2 .\end{cases}
$$

Now we are going to discuss how to find the optimal action for each state. For $\mathbf{s} \in \mathbf{S}$, we define $V(\mathbf{s})$ as the expected energy consumption for the system to evolve from $\mathbf{s}$ to $\mathbf{s}_{\mathrm{g}}$ given that optimal actions are chosen in every state, and define $\pi(\mathbf{s})$ as the optimal action at state $\mathbf{s}$. It is clear that $V(\mathbf{s})$ is non-zero except when $\mathbf{s}=\mathbf{s}_{\mathrm{g}}$. For $\mathbf{s} \in \mathbf{S} \backslash\left\{\mathbf{s}_{\mathrm{g}}\right\}$, we define $V(\mathbf{s}, a)$ as the expected energy consumption for the system to evolve from $\mathbf{s}$ to $\mathbf{s}_{\mathrm{g}}$ given that action $a$ is chosen for state $\mathbf{s}$ and optimal actions are chosen for the other states. For a given action $a=\left(b, \theta_{m}\right) \in \mathbf{A}, V(\mathbf{s}, a)$ can be computed by solving the following equation:

$$
V(\mathbf{s}, a)=\theta_{m}+\sum_{\mathbf{s}^{\prime} \in \mathbf{S} \backslash\{\mathbf{s}\}} p\left(\mathbf{s}^{\prime} \mid \mathbf{s}, a\right) V\left(\mathbf{s}^{\prime}\right)+p(\mathbf{s} \mid \mathbf{s}, a) V(\mathbf{s}, a),
$$

where $\theta_{m}$ means that each state transition takes one transmission and $\theta_{m}$ is the energy consumption of that transmission. Note that the summation only involves those states that can be immediately reached from $\mathbf{s}$. In other words, state $\mathbf{s}^{\prime}$ is involved only when $p\left(\mathbf{s}^{\prime} \mid \mathbf{s}, a\right)$ is non-zero. Equation (2) can be written as:

$$
V(\mathbf{s}, a)=\left[\theta_{m}+\sum_{\mathbf{s}^{\prime} \in \mathbf{S} \backslash\{\mathbf{s}\}} p\left(\mathbf{s}^{\prime} \mid \mathbf{s}, a\right) V\left(\mathbf{s}^{\prime}\right)\right] /[1-p(\mathbf{s} \mid \mathbf{s}, a)]
$$

Note that our problem has the property that the state-transition graph is a directed acyclic graph. It mea-ns that given two distinct states, $\mathbf{s}$ and $\mathbf{s}^{\prime}$, if $\mathbf{s}^{\prime}$ can be reached from $\mathbf{s}$ after a certain number of state transitions, then $\mathbf{s}$ cannot be reached from s'. Figure 2 shows an example of the state transition graph for $K=2$ and $N=2$. For directed acyclic graph, it is well known that there is a topological ordering of vertices, which means that if there is a state transition from $\mathbf{s}$ to $\mathbf{s}^{\prime}$, then $\mathbf{s}$ comes before $\mathbf{s}$ ' in the ordering. Due to this property, $V(\mathbf{s})$ can be computed backward from $V\left(\mathbf{s}_{\mathrm{g}}\right)$. To compute $V(\mathbf{s})$, we apply the formula in (3) to find $V(\mathbf{s}, a) . V(\mathbf{s})$ and the optimal action at $\mathbf{s}$ can then be obtained by

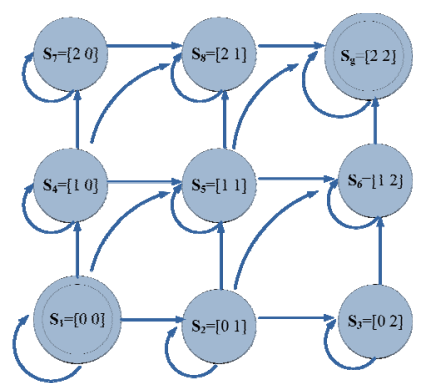

Figure 2. State-transition graph for $K=2$ and $N=2$.

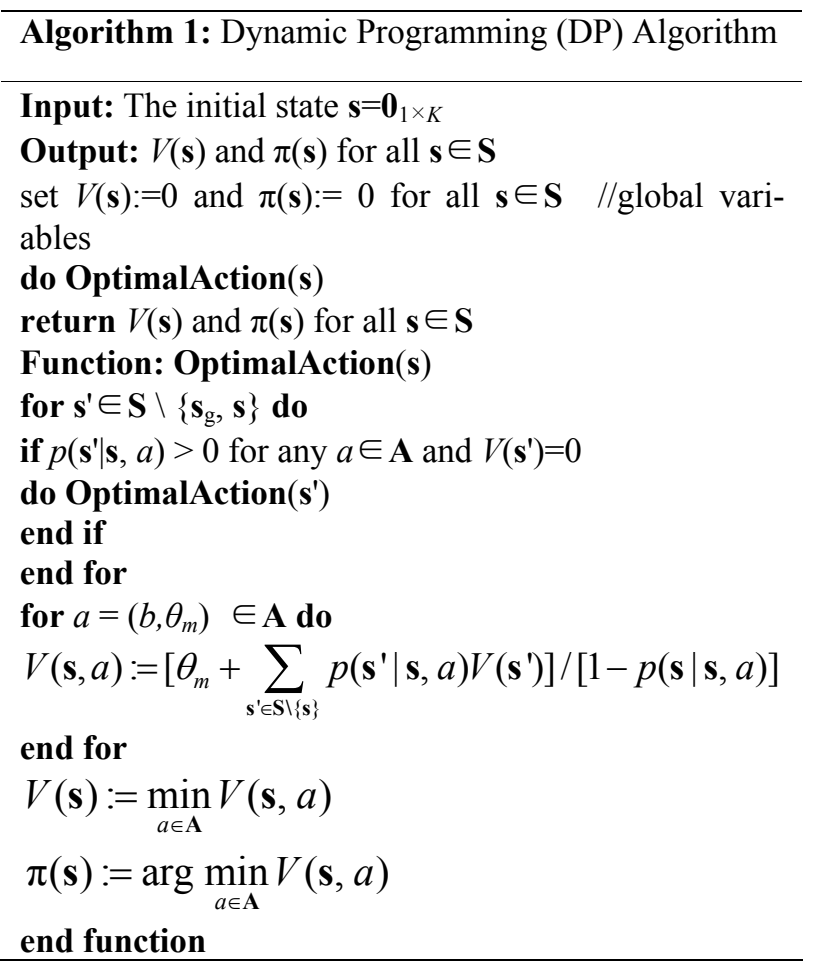

$$
V(\mathbf{s})=\min _{a \in \mathbf{A}} V(\mathbf{s}, a) \text { and } \pi(\mathbf{s})=\arg \min _{a \in \mathbf{A}} V(\mathbf{s}, a),
$$

respectively. To implement this idea, recursion may be used, and we state the recursive algorithm in Algorithm (1). We call it Dynamic Programming (DP) scheme.

By the principle of optimality [12], DP is optimal in terms of minimizing expected energy consumption.

\section{Heuristic Schemes}

While DP is optimal, its time complexity grows exponentially with $K$. To reduce complexity, we propose two fast heuristic algorithms for large networks. When the number of users is relatively small, we propose a greedy algorithm which dynamically changes the transmit power. When the number of users is very large, we propose an algorithm which uses fixed transmit power.

\subsection{Greedy Algorithm}

The greedy algorithm has the following three steps.

Step 1: At the beginning of a time slot, let $s_{j}$, where 0 $<j \leq K$, denote the number of packets that $U_{j}$ has received. We first choose the user $U_{i}$ which has received the least number of packets. If there are multiple qualified users, we will randomly pick one of them.

Step 2: Select the base station $B S_{b}{ }^{*}$ that is closer to $U_{i}$ as the sender. If both $B S_{1}$ and $B S_{2}$ have the same distance from $U_{i}$, randomly pick one of them as the sender in the current slot.

Step 3: We define a parameter $E_{b i}\left(\theta_{m}\right)$ for each trans- 
mit power level $\theta_{m}$. At a particular slot, $E_{b i}\left(\theta_{m}\right)$ is defined as the expected energy consumption required to make user $U_{i}$ successfully receive a packet, assuming that $B S_{b}$ is selected to transmit with power $\theta_{m} . E_{b i}\left(\theta_{m}\right)$ can be computed as follows.

$$
E_{b i}\left(\theta_{m}\right)=\frac{1}{R_{b i}\left(\theta_{m}\right)} \theta_{m},
$$

where $R_{b i}\left(\theta_{m}\right)$ is the reliability of the channel from $B S_{b}$ to $U_{i}$ with transmit power $\theta_{m}$.

After computing the $E_{b i}\left(\theta_{m}\right)$ value for each $\theta_{m}$, choose the transmit power $\theta_{m}{ }^{*}$ that corresponds to the minimal $E_{b i}\left(\theta_{m}\right)$ as the transmit power of current slot. If there exists more than one $E_{b i}\left(\theta_{m}\right)$ which has the minimal value, just randomly pick one of them. Then $B S_{b}{ }^{*}$ will broadcast a packet to all users with transmit power $\theta_{m} *$.

Then go back to Step 1 and repeat the procedure until all the users are complete. The greedy algorithm can be summarized as Algorithm (2), which is called Greedy Transmission (GT) algorithm.

\subsection{Fixed Power Algorithm}

When the number of users is very large, the users with worst channels locate at the boundaries of the cells with high probabilities. For such scenarios, we propose the following algorithm with fixed transmit power.

$B S_{1}$ transmits at odd time slots and $B S_{2}$ transmits at even slots. The transmit power is always set to a fixed power level $\theta^{*} . B S_{1}$ and $B S_{2}$ take turns to transmit until all users are complete.

The transmit power $\theta^{*}$ is determined as follows. Consider a wireless system which consists of $B S_{1}$ and only one user $U_{1}$. Suppose $U_{1}$ is located at the border of the cell, i.e. the distance between $U_{1}$ and $B S_{1}$ is the radius $r$. For each $\theta_{m}$, we calculate the expected energy consumption $E_{11}\left(\theta_{m}\right)$ defined by Equation (4). Then select the power level with minimal $E_{11}\left(\theta_{m}\right)$ as $\theta^{*}$, which means $\theta^{*}$ is the optimal power level for $U_{1}$, a user locating at the boundary.

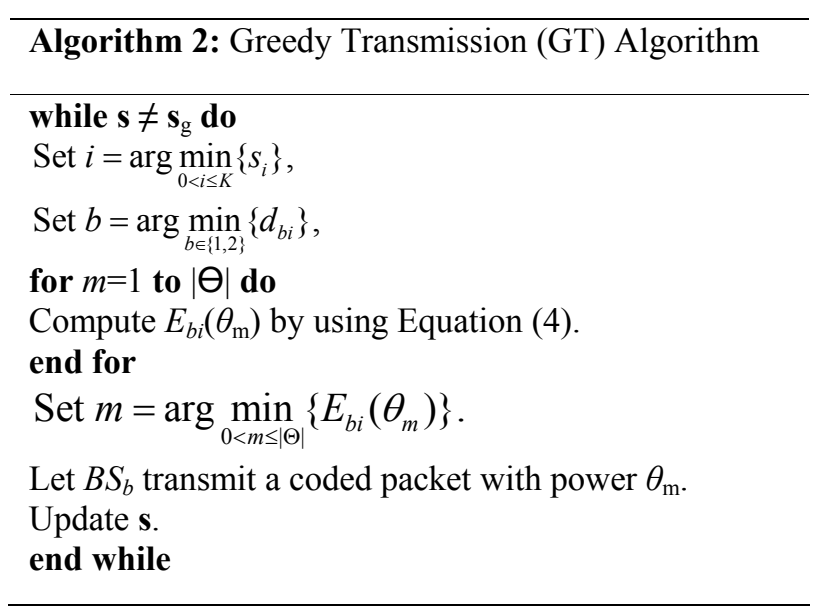

Note that this algorithm has such a feature that it does not require any channel state information of users. We call this algorithm the Fixed Power (FP) algorithm.

\section{Numerical Results}

We evaluate the performance of proposed algorithms via simulations. The downlink channels are modeled containing combined effects of path loss and Rayleigh fading. The minimal transmit power $\theta_{\min }$ and maximal transmit power $\theta_{\max }$ are set to $100 \mathrm{~mW}(20 \mathrm{dBm})$ and $20 \mathrm{~W}$ (about $43 \mathrm{dBm}$ ), respectively. $\Theta$ includes 20 ordered power levels which are linearly spaced between and including $\theta_{\min }$ and $\theta_{\max }$. The SINR threshold $\Gamma$ is set to $3 \mathrm{~dB}$. $\delta$ is set to different values to evaluate the performances under different cell locations. Other parameters are shown in Table 1 [13]. For each set of parameters, the results are averaged over 10,000 repetitions.

We compare the proposed algorithms with the scheme named NCPC in [5] and an ARQ scheme. For NCPC, we set the parameter, the extension radius of the center cell, to $1.15 r$, which is the same as the simulation in [5]. We refer the reader to [5] for details of NCPC. In the ARQ scheme, $B S_{1}$ transmits at odd slots and $B S_{2}$ transmits at even slots. Note that if all the users in a cell are complete, only the base station of the other cell will transmit at the remaining time slots. In each cell, the base station retransmits each uncoded packet until all the users in its cell has received it. The transmit power is determined as follows. At a particular slot, the base station $B S_{b}$ first selects a target user $U_{i}$ which requires the packet to be broadcast while has the longest distance from the base station. Then, use Equation (4) to calculate the expected energy consumption $E_{b i}\left(\theta_{m}\right)$ for each $\theta_{m}$. Finally, select the power level with minimal $E_{b i}\left(\theta_{m}\right)$ as the transmit power in that slot.

For small scale networks, we compare the performances of DP, GT and FP with NCPC and ARQ. In Figure 3, we first evaluate the performances of different cell locations with $N=4$ and $K=4$ and $\delta$ varies from 0.3 to 1.9. When $\delta=0.3$, the two cells are almost totally overlapped with each other. When $\delta=1.9$, the two cells are almost separated. We can find that both DP and GT always perform better than NCPC and ARQ. In particular,

Table 1. Simulation parameters[13].

\begin{tabular}{ll}
\hline \multicolumn{1}{c}{ Parameter } & \multicolumn{1}{c}{ Value } \\
\hline Cell radius $r$ & $1 \mathrm{Km}$ \\
Bandwidth & $20 \mathrm{MHz}$ \\
No. of resource blocks & 100 \\
BS antenna gain & $11 \mathrm{dBi}$ \\
MS antenna gain & $0 \mathrm{dBi}$ \\
Minimum coupling loss & $53 \mathrm{~dB}$ \\
Path loss & $128.1+37.6 \log _{10}\left(d_{b i}\right), d_{b i}$ in $\mathrm{Km}$ \\
Noise density & $-165 \mathrm{dBm} / \mathrm{Hz}$ \\
Noise figure & $9 \mathrm{~dB}$ \\
\hline
\end{tabular}




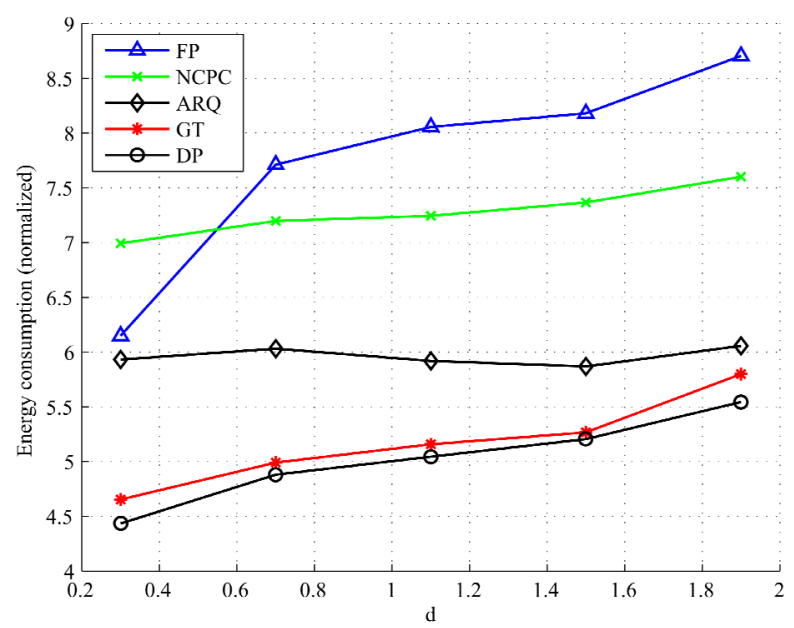

Figure 3. Energy consumption for $N=4, K=4$ and various $\delta$.

compared with NCPC when $\delta=0.3$, DP and GT can reduce energy consumption by $37 \%$ and $34 \%$, respectively. Compared with ARQ, when $\delta=0.3$, DP and GT can reduce energy consumption by $26 \%$ and $22 \%$, respectively. To investigate the effects of number of users, we run simulations with $N=4, \delta=1.0$ and $K$ varies from 2 to 4 . The simulation results are shown in Figure 4. DP and GT also outweigh NCPC and ARQ in all the scenarios. Especially, compared with NCPC, both DP and GT can reduce energy consumption by about $50 \%$ when $K=2$. Compared with ARQ, when $K=4$, DP and GT can reduce energy consumption by $17 \%$ and $16 \%$, respectively. In both Figure 3 and Figure 4, the performance gap between DP and GT is less than 6\%, which means the GT performs very well and is close to the optimal scheme. In both Figure 3 and Figure 4, FP performs worse than other schemes. The reason is that, when the number of users is small, the "worst" user is not located near the cell boundary with high probabilities, where the worst user means it has the longest distance from base station. That means the transmit power of FP is too large and FP is energy inefficient.

For large scale networks, we evaluate the performance of GT, FT, NCPC and ARQ with changing $K$ and $\delta$. Considering the computational complexity of DP, it is not taken into comparison. In Figure 5, the parameters are set to $N=60, K=50$ and $\delta$ varies from 0.3 to 1.9. Both GT and FP perform better than NCPC and ARQ, no matter how the cells are located. In particular, compared with NCPC, when $\delta=0.3$, GT and FP can reduce energy consumption by $19 \%$ and $15 \%$, respectively. Compared with ARQ, when $\delta=1.9$, GT and FP can reduce energy consumption by $50 \%$ and $47 \%$, respectively. We also evaluate the performances with $N=60, \delta=1$ and $K$ varies from 20 to 100. The simulation results are shown in Figure 6. Both GT and FP also require less transmit energy. In particular, compared with NCPC, when $K=100$,
GT and FP can reduce energy consumption by $18 \%$ and $14 \%$, respectively. Compared with ARQ, when $K=100$, GT and FP can reduce energy consumption by $57 \%$ and $55 \%$, respectively. In both Figure 5 and Figure 6, the performance of FP is comparable to that of GT. In Figure 6, the gap is less than $5 \%$ when $K \geq 40$. As the number of users increases, the gap between GT and FP becomes smaller and smaller. The reason is that, when number of users is large, the users with worst channels are located near the cell boundary. In that case, the transmit power selected by GT is close, or even equal, to that selected by FP. Therefore, their performances are very close.

\section{Conclusions}

In this paper, we consider the problem of minimizing the expected transmit energy consumption for a two-cell broadcasting system. On one hand, we apply the innovative network coding to reduce the number of retransmissions. On the other hand, we use power control to control

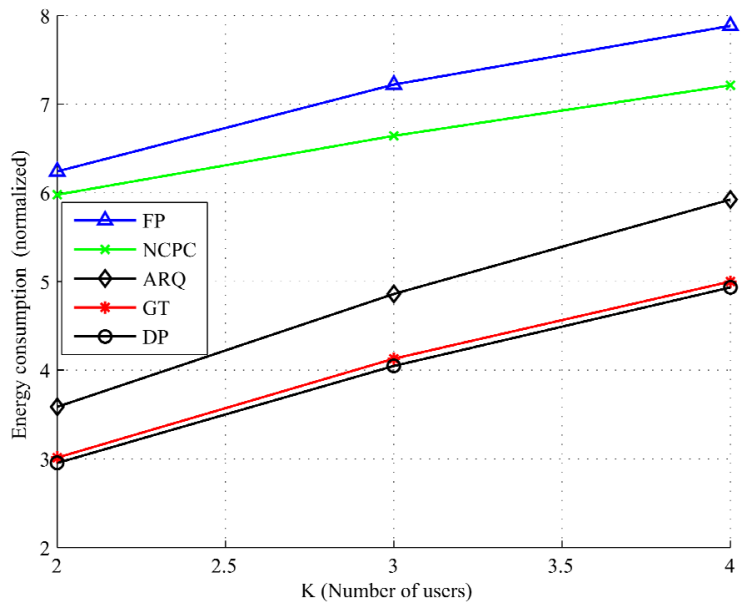

Figure 4. Energy consumption for $N=4, \delta=1$ and various $K$.

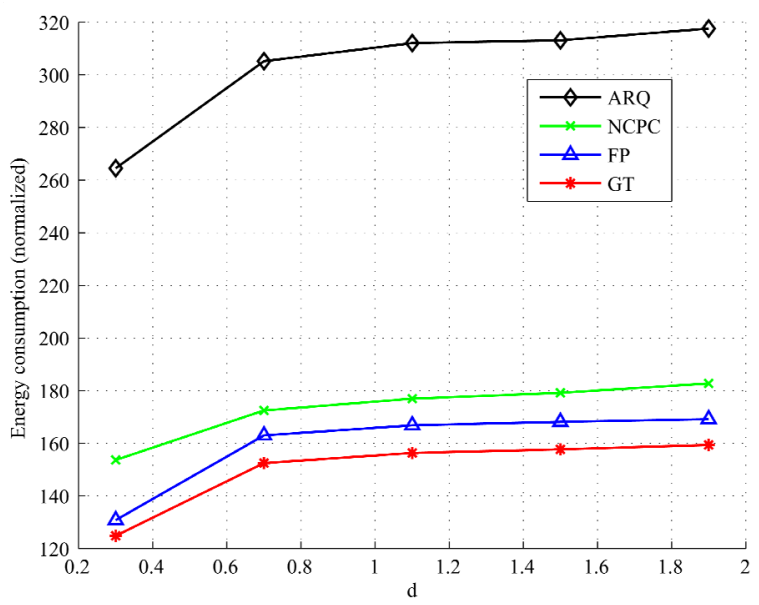

Figure 5. Energy consumption for $N=60, K=50$ and various $\delta$. 


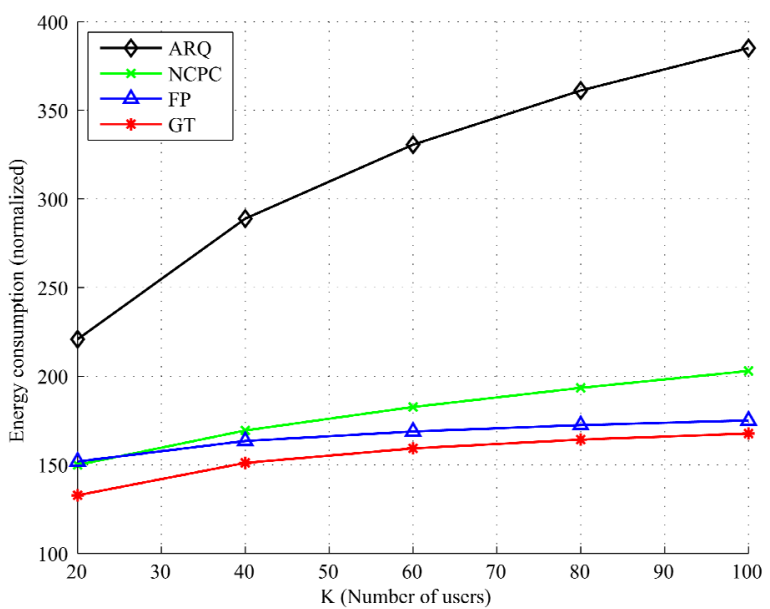

Figure 6. Energy consumption for $N=60, \delta=1$ and various $K$.

the received SINR such that the transmit efficiency can be improved. Besides, we also consider the scheduling problem of the two base stations. We first propose an optimal algorithm called DP, in the sense of minimizing expected energy consumption, which uses dynamic programming to choose the optimal action for each state. To reduce complexity, we also propose two sub-optimal greedy algorithms, called GT and FP, respectively, for large scale networks. Simulation shows that GT has a very close performance to that of the optimal DP when the network is small. When the number of users is very large, both FP and GT outweigh NCPC and ARQ. Although the algorithms are designed for two-cell systems in this paper, they can be extended to multiple-cell systems.

\section{Acknowledgements}

The work described in this paper was partially supported by a grant from the University Grants Committee of the Hong Kong Special Administrative Region, China (Project No. AoE/E-02/08).

Part of this work was done when the first author visited Yonsei University in 2012 Fall.

\section{REFERENCES}

[1] S. Vadgama, "Trends in Green Wireless Access," FU-
JITSU Scientific \& Technical Journal, Vol. 45, 2009, pp. 404-408.

[2] S. Zeadally, S. U. Khan and N. Chilamkurti, "Energy-Efficient Networking: Past, Present, and Future," The Journal of Supercomputing, Vol. 62, No. 3, 2012, pp. 1093-1118. doi:10.1007/s11227-011-0632-2

[3] Ericsson, "Sustainable Energy Use in Mobile Communications," White paper, Aug. 2007.

[4] S. Y. R. Li, R. W. Yeung and N. Cai, "Linear Network Coding," IEEE Transactions on Information Theory, Jun. 2003, pp. 371-381. doi:10.1109/TIT.2002.807285

[5] T. Tran, D. Nguyen, T. Nguyen and D. Tran, "Joint Network Coding and Power Control for Cellular Radio Networks," Proceedings of the Second International Conference on Communications and Electronics, Jun. 2008, pp. 109 - 114 .

[6] L. Lu, F. Sun, M. Xiao and L. K. Rasmussen, "Relay-Aided Multi-cell Broadcasting with Random Network Coding," Proceedings of International Symposium on Information Theory and its Applications, Dec. 2010, pp. 957-962.

[7] H. Y. Kwan, K. W. Shum and C. W. Sung, "Generation of Innovative and Sparse Encoding Vectors for Broadcast Systems with Feedback," Proceedings of IEEE International Symposium on Information Theory, Saint Petersburg, Russia, Aug. 2011, pp. 1161 - 1165.

[8] H. Y. Kwan, K. W. Shum and C. W. Sung, "Linear Network Code for Erasure Broadcast Channel with Feedback: Complexity and Algorithms," arXiv: 1205.5324v1, May 2012.

[9] M. Chiang, P. Hande, T. Lan and W. Tan, "Power Control in Wireless Cellular Networks," Foundations and Trends in Networking, Vol. 2, No. 4, 2008, pp. 381-533. doi:10.1561/1300000009

[10] A. Sridhar and A. Ephremides, "Energy Optimization in Wireless Broadcasting through Power Control," Ad Hoc Networks, Vol. 6, 2008, pp. 155-167. doi:10.1016/j.adhoc.2006.11.001

[11] M. K. Simon and M. S. Alouini, "Fading Channel Characterization and Modeling," Digital Communication over Fading Channels A Unified Approach to Performance Analysis, 2nd ed., Wiley, 2005.

[12] D. P. Bertsekas, "The Dynamic Programming Algorithm," Dynamic Programming and Optimal Control, 3nd ed., Mass.: Athena Scientific, 2005.

[13] 3GPP TR 25.942 v9.0, RF System Scenarios, Dec. 2009. 